QMW-PH-93-4

hep-ph/9303250

\title{
DOMAIN WALL FORMATION IN THE POST-INFLATIONARY UNIVERSE
}

\author{
ZYGMUNT LALAK凹 \\ Department of Physics \\ Theoretical Physics \\ 1 Keble Road \\ Oxford OX1 3NP \\ U.K. \\ and \\ STEVEN THOMAS \\ Department of Physics \\ Queen Mary and Westfield College \\ Mile End Road \\ London E1 \\ U.K.
}

\begin{abstract}
We consider the evolution of the probability distribution $\mathrm{P}(\chi, \bar{\chi}, \mathrm{t})$, associated with an inhomogeneous light scalar field $\chi$ in the Robertson-Walker Universe, where the inhomogeneities are produced by quantum fluctuations during an earlier inflationary epoch. For a specific choice of scalar potential which occurs in models of so called late-time phase transitions in which domain walls are produced, $\mathrm{P}$ is shown to evolve from a Gaussian to a non-Gaussian distribution. The structure of the latter justifies

\footnotetext{
${ }^{1}$ On leave from the Institute of Theoretical Physics, University of Warsaw. *BITNET: lalak@dionysos.thphys.ox.ac.uk; thomas@v2.ph.qmw.ac.uk
} 
the recent use of 3-dimensional percolation theory to describe the initial distribution of domain walls in these models. 


\section{Introduction}

The idea that large scale structure in the Universe might appear as the result of a 'late-time' phase transition associated with an extremely light scalar field, has been the subject of a number of recent papers $[1,2]$. Large scale structure in these models could be generated either by density perturbations due to fluctuations in this scalar field, or because of the gravitational attraction of topological defects such as domain walls that might be produced. If the walls form a network, then in order to avoid generating too large a microwave background anisotropy the phase transition should occur after photon decoupling. (Alternatively, the phase transition could occur prior to this time and still be consistent with observations of the microwave background if, for example, the domain walls that form are isolated 'bags' that subtend small angular distances on the sky.) For the phase transition to occur after photon decoupling requires the mass of the scalar to be extremely small, of order $10^{-29} \mathrm{eV}$, and for it to have very weak interactions [1]. Although masses of this size might at first sight appear to be unnatural, they have been shown to occur in several particle physics scenarios $[2,3]$.

It should be understood that by 'phase transition' we do not necessarily mean one which occurs at finite temperature, which would normally require the scalar field responsible for wall formation to be in thermal equilibrium. Since we are dealing with a weakly interacting field, this latter requirement might be problematic (but see ref.[1] for a further discussion of this point.) In fact, the fluctuations in the scalar field that eventually produce the walls need not be thermal in origin, but might be the result of quantum fluctuations during an earlier inflationary epoch [4]. This is the viewpoint we shall adopt in this paper.

It has been known for some time [5,6], that significant clustering of galaxies occurs at length scales of order 1Mpc. Moreover, detailed redshift surveys [7], have revealed the presence of walls, voids and other gravitational attractors on scales up to 50Mpcs, and at redshifts $\mathrm{z}<5$ which corresponds to relatively recent times in the Universe's evolution. It is possible that such clustering might be explained by primordial fluctuations alone, such as those produced during an earlier inflationary epoch. (In fact, such primordial perturbations are at least consistent with the recent discovery of a microwave background anisotropy [8], $\left.\delta \mathrm{T} / \mathrm{T} \approx 6 \times 10^{-6}\right)$. However, it is difficult for such fluctuations to simultaneously account for structure on both large and small scales. A 
better approach might be a kind of hybrid model containing primordial density perturbations plus those due to the formation of late-time domain walls, where the latter would describe the kind of structures discovered by the redshift survey [7].

In refs.[3,9], a detailed analysis of the distribution of domain walls in such a model was presented, where the walls are formed by quantum fluctuations in the scalar field during an earlier inflationary epoch. Even though these fluctuations arise from inflation, they do not generate walls until much later on, when inflation ends and the Robertson-Walker (R-W) phase begins. (The idea that domain walls can be produced in this way was first investigated, in a different context, in ref.[4]). An important assumption of the analysis in refs. [3,9], was that 3-dimensional percolation theory [10], provides a good description of the domain wall network immediately after their formation. The criterion for percolation theory to be strictly applicable, is that the scalar field $\chi$ describing the walls must be in either of two degenerate minima in all causally connected regions of space, with probability $\wp$ and (1- $\wp)$ respectively. By contrast, the probability distribution $\mathrm{P}(\chi, \bar{\chi})$ of $\chi$ immediately after inflation ends is a Gaussian centred about the value $\bar{\chi}$. Since $\mathrm{P}(\chi, \bar{\chi})$ is the distribution of possible $\chi$ values throughout space, it is clear that we have to do more to meet the above criterion.

The purpose of this letter is to justify the use of percolation theory by showing that the distribution $\mathrm{P}(\chi, \bar{\chi})$ evolves during the $\mathrm{R}-\mathrm{W}$ phase to a non-Gaussian distribution whose maxima approach two degenerate minima of the scalar potential. In this way the new distribution for $\chi$ comes much closer to satisfying the percolation criterion discussed above.

The paper is organized as follows : in section 2 we briefly review some concepts concerning scalar field quantization in de Sitter space and the appearance of the distribution $\mathrm{P}(\chi, \bar{\chi})$. In section 3 , we show how $\mathrm{P}(\chi, \bar{\chi})$ evolves into a non-Gaussian distribution and illustrate this with various numerical plots.

\section{Scalar field fluctuations in de Sitter space}

In this section we will briefly review some aspects of scalar field quantization in de Sitter space, and explain how this leads to the production of an inhomogeneous quasiclassical field. The reader is referred to refs.[11] for details. First consider quantization

of a scalar field $\phi$ in the de Sitter geometry $\mathrm{ds}^{2}=\mathrm{dt}^{2}-a^{2}(\mathrm{t}) \mathrm{dx}^{2}$, where $\mathrm{x}$ and $\mathrm{t}$ are 
comoving coordinates. The scale factor $a(\mathrm{t})=\mathrm{e}^{\mathrm{H}_{i} \mathrm{t}}$, and $\mathrm{H}_{i}$ is Hubble's constant during inflation. I The equation of motion for $\phi$ is

$$
\ddot{\phi}-a^{-2}(\mathrm{t}) \nabla^{2} \phi+3 \mathrm{H}_{i} \dot{\phi}+\frac{\mathrm{dV}}{\mathrm{d} \phi}=0
$$

Note that we choose the time variable $t=\tilde{t}-\tilde{t}_{i}$ where $\tilde{t}$ is actual cosmological time lapsed, and $\tilde{t}_{i}$ is the time at which inflation begins. The fourier decomposition of $\phi$ is

$$
\phi(\mathrm{x}, \mathrm{t})=\int \frac{\mathrm{d}^{3} \mathrm{p}}{(2 \pi)^{3 / 2}}\left\{a_{\mathrm{p}} \mathrm{e}^{i \mathrm{p} \cdot \mathrm{x}} \phi_{\mathrm{p}}(\mathrm{t})+\text { h.c }\right\}
$$

where $\mathrm{p}$ is the comoving 3-momentum. The modes $\phi_{\mathrm{p}}(\mathrm{t})$ are given in terms of Hankel functions [11], whose explicit form we shall not need in this paper, and $a_{\mathrm{p}},\left(a_{\mathrm{p}}^{\dagger}\right)$ are anihilation (creation) operators respectively. In an infinite de Sitter space, the integration over 3-momenta $\mathrm{p}$ would continue down to $\mathrm{p}=0$. However, in the inflationary Universe there is the inflationary horizon whose comoving size $\mathrm{H}_{i}^{-1}$, acts as an infrared cutoff on the spectrum.

Next we split $\phi$ into two parts, one which includes averaging over modes with physical momentum $\mathrm{k} \equiv \mathrm{p} \exp \left(-\mathrm{H}_{\mathrm{i}} \mathrm{t}\right)>\mathrm{H}_{\mathrm{i}}$ (where $\mathrm{H}_{i}$ is the physical distance to the horizon at the start of inflation), and the other a kind of coarse grained field smoothed out over horizon size scales,

$$
\begin{aligned}
\phi(\mathrm{x}, \mathrm{t}) & =\int \frac{\mathrm{d}^{3} \mathrm{p}}{(2 \pi)^{3 / 2}} \Theta\left(\mathrm{p}-\epsilon \mathrm{H}_{\mathrm{i}} \mathrm{e}^{\mathrm{H}_{\mathrm{i}} \mathrm{t}}\right)\left\{\mathrm{a}_{\mathrm{p}} \phi_{\mathrm{p}}(\mathrm{t}) \mathrm{e}^{\mathrm{ip} \cdot \mathrm{x}}+\mathrm{h} . \mathrm{c}\right\} \\
& +\chi(\mathrm{x}, \mathrm{t})-\bar{\chi}
\end{aligned}
$$

In eq.(3) $\bar{\chi}$ is the mean value of the coarse grained field $\chi$ during inflation, whose value we shall discuss later. $\epsilon$ is a small parameter $0<\epsilon \ll 1$, which ensures that the 'filter' function represented by the step function in eq.(3) rapidly vanishes for comoving momenta $\mathrm{p} \approx \mathrm{H}_{i} \mathrm{e}^{\mathrm{H}_{i} \mathrm{t}}[11]$. By solving the equations of motion for the modes $\phi_{\mathrm{p}}$, in the linearized approximation, one may deduce that slowly varying fields $\chi$ satisfy a Langevin type equation,

$$
\dot{\chi}(\mathrm{x}, \mathrm{t})=\mathrm{f}(\mathrm{x}, \mathrm{t})+\left(\frac{1}{3 \mathrm{H}_{i}} \mathrm{e}^{-2 \mathrm{H}_{i} \mathrm{t}} \nabla^{2} \chi-\frac{\mathrm{dV}}{\mathrm{d} \chi}\right)
$$

\footnotetext{
${ }^{2}$ Here we assume that the field responsible for inflation is not $\phi$, but some other field in another sector of the theory.
} 
where the noise $f(x, t)$ is given by

$$
\mathrm{f}(\mathrm{x}, \mathrm{t})=\int \frac{\mathrm{d}^{3} \mathrm{p}}{(2 \pi)^{3 / 2}} \delta\left(\mathrm{p}-\epsilon \mathrm{e}^{\mathrm{H}_{\mathrm{i}} \mathrm{t}} \mathrm{H}_{\mathrm{i}}\right) \epsilon \mathrm{e}^{\mathrm{H}_{\mathrm{i}} \mathrm{t}} \mathrm{H}_{\mathrm{i}}{ }^{2}\left\{\mathrm{a}_{\mathrm{p}} \phi_{\mathrm{p}}(\mathrm{t}) \mathrm{e}^{\mathrm{ip} \cdot \mathrm{x}}+\text { h.c }\right\}
$$

Since [11], one can also prove that the noise $f(x, t)$ satisfies $\langle f(x, t) f(x, t)\rangle=$ $\left(\mathrm{H}_{i}{ }^{3} / 4 \pi^{2}\right) \delta(\mathrm{t}-\mathrm{t})$, one may derive a Fokker-Planck $(\mathrm{F}-\mathrm{P})$ equation from the Langevin equation (4). Introducing the normalized probability distribution $\mathrm{P}(\chi, \bar{\chi}, \mathrm{t})$ for the field $\chi$

$$
\langle\mathrm{F}(\chi(\mathrm{x}, \mathrm{t})-\bar{\chi})\rangle_{\mathrm{f}} \equiv \int_{-\infty}^{+\infty} \mathrm{d} \chi \mathrm{F}(\chi-\bar{\chi}) \mathrm{P}(\chi, \bar{\chi}, \mathrm{t})
$$

where $\mathrm{F}$ is an arbitrary function, and \langle\rangle$_{\mathrm{f}}$ signifies averaging with respect to the noise $\mathrm{f}$ defined earlier, the corresponding F-P equation takes the form

$$
\frac{\partial \mathrm{P}(\chi, \bar{\chi}, \mathrm{t})}{\partial \mathrm{t}}=\frac{\partial}{\partial \chi}\left(\frac{1}{3 \mathrm{H}_{i}} \frac{\partial \mathrm{V}}{\partial \chi} \mathrm{P}(\chi, \bar{\chi}, \mathrm{t})\right)+\frac{\mathrm{H}_{i}{ }^{3}}{8 \pi^{2}} \frac{\partial^{2} \mathrm{P}(\chi, \bar{\chi}, \mathrm{t})}{\partial \chi^{2}}
$$

The boundary condition on $\mathrm{P}(\chi, \bar{\chi}, \mathrm{t})$ at $\mathrm{t}=0\left(\tilde{\mathrm{t}}=\tilde{\mathrm{t}}_{0}\right)$, the start of inflation, is taken to be $\mathrm{P}(\chi, \bar{\chi}, \mathrm{t})=\delta(\chi-\bar{\chi})$. With this initial condition, $\mathrm{P}(\chi, \bar{\chi}, \mathrm{t})$ for $\mathrm{t}>0$ can be interpreted as the fraction of the initial comoving volume occupied by the coarse grained field $\chi$ at time t.

We are interested in scalar field potentials of the form

$$
\mathrm{V}(\chi)=\mathrm{m}_{\chi}^{2} \mathrm{M}^{2}\left[\cos \left(\frac{\chi}{\mathrm{M}}\right)+1\right]
$$

which occur in models describing light domain walls [1]. Since in these models the mass scale $\mathrm{M} \approx \mathrm{H}_{i}$, whilst $\mathrm{m}_{\chi} \ll \mathrm{M}$ we suspect that solution for the F.P. eq. (7) is well approximated by the solution to the equation with $\mathrm{V}=0$. To see this, (following the derivations given in [11] for general V), we can obtain an approximate solution to the F-P equation, with $\mathrm{V}$ given in eq. (8) as

$$
\mathrm{P}(\chi, \bar{\chi}, \mathrm{t})=\frac{\sqrt{2 \pi}}{\mathrm{M} \Delta(\tau)} \exp \left(-\frac{(\chi-\bar{\chi})^{2}}{2 \mathrm{M}^{2} \Delta^{2}(\tau)}\right)
$$

with $\tau \equiv \mathrm{Mt} / 4 \pi$, and where the dispersion $\Delta^{2}(\tau)$ satisfies

$$
\frac{\mathrm{d} \Delta^{2}(\tau)}{\mathrm{d} \tau}=\frac{8 \pi \mathrm{m}_{\chi}^{2}}{3 \mathrm{hM}^{2}} \cos \overline{\mathrm{x}}(\tau) \Delta^{2}(\tau)+\frac{\mathrm{h}^{3}}{\pi}
$$

In eq. (10) $\mathrm{h} \equiv \mathrm{H}_{i} / \mathrm{M}$, and $\overline{\mathrm{x}}(\tau)$ is an intermediate variable satisfying 


$$
\dot{\overline{\mathrm{x}}}(\tau)=\frac{4 \pi \mathrm{m}_{\chi}^{2}}{3 \mathrm{hM}^{2}} \operatorname{sinx}(\tau)
$$

The point is that from the solution to eq.(11), i.e.

$$
\overline{\mathrm{x}}(\tau)=2 \tan ^{-1}\left(\tan \left(\frac{\mathrm{x}_{0}}{2}\right) \exp \left(\frac{4 \pi \tau \mathrm{m}_{\chi}^{2}}{3 \mathrm{hM}^{2}}\right)\right)
$$

we see that since the ratio $\mathrm{m}_{\chi} / \mathrm{M} \ll 1[1], \mathrm{x}(\tau) \approx \mathrm{x}_{0}$, for all relevant time scales during inflation, where $\mathrm{x}_{0}$ is a constant of integration. This allows one to solve eq.(10) directly for the dispersion

$$
\Delta^{2}(\tau)=\frac{3 \mathrm{~h}^{4} \mathrm{M}^{2}}{8 \pi^{2} \mathrm{~m}_{\chi}^{2} \cos \left(\mathrm{x}_{0}\right)}\left(\exp \left[\frac{8 \pi \mathrm{m}_{\chi}^{2}}{3 \mathrm{hM}^{2}} \cos \left(\mathrm{x}_{0}\right) \tau\right]-1\right)
$$

Here $\Delta(\tau)$ satisfies $\Delta(\tau=0)=0$ corresponding to our previous boundary condition $\mathrm{P}(\chi, \bar{\chi}, \mathrm{t})=\delta(\chi-\bar{\chi})$ at $\mathrm{t}=0$. However, again we see the very small factors of $\mathrm{m}_{\chi}{ }^{2} / \mathrm{M}^{2}$ in the exponent of eq.(13), so we can expand and keep lowest order terms only. This gives

$$
\Delta^{2}(\tau) \approx \frac{\mathrm{h}^{3}}{\pi} \tau+\mathrm{O}\left(\frac{\mathrm{m}_{\chi}^{2}}{\mathrm{M}^{2}}\right)
$$

Hence, for the scalar potentials of relevance to theories describing light domain walls, it is a very good approximation to take as the solution to the F-P equation (7) the usual Gaussian probability distribution

$$
\mathrm{P}(\chi, \bar{\chi}, \mathrm{t})=\sqrt{\frac{8 \pi^{3}}{\mathrm{H}_{i}{ }^{3} \mathrm{t}}} \exp \left(-\frac{2 \pi^{2}(\chi-\bar{\chi})^{2}}{\mathrm{H}_{i}{ }^{3} \mathrm{t}}\right)
$$

centred about $\chi=\bar{\chi}$.

Thus for example the correlation of $\chi(\mathrm{x}, \mathrm{t})$ with itself is

$$
\langle(\chi(\mathrm{x}, \mathrm{t})-\bar{\chi})(\chi(\mathrm{x}, \mathrm{t})-\bar{\chi})\rangle=\frac{\mathrm{H}_{i}{ }^{3} \mathrm{t}}{4 \pi^{2}}+\mathrm{O}\left(\left(\frac{\mathrm{m}_{\chi}}{\mathrm{M}}\right)^{2}\right)
$$

Even if one had considered the correlation function in eq.(16) at two separate (comoving) points $\mathrm{x}, \mathrm{y}$ the r.h.s. is typically only small at extremely large physical separations $|\mathrm{x}-\mathrm{y}| \exp \mathrm{H}_{i} \mathrm{t} \sim \mathrm{H}_{i}{ }^{-1} \exp \mathrm{H}_{i} \mathrm{t}$. Because the physical correlation length of $\chi$ is thus typically of order the inflationary horizon, $\chi$ can be interpreted as a quasi-classical field on very much smaller distance scales [11] . 


\section{Evolution of $\mathrm{P}(\chi, \bar{\chi})$ to a non- Gaussian probability distribution.}

As we have indicated above and as is explained in ref.[4], the fluctuations about the mean value of $\chi$ give rise to an inhomogeneous quasi-classical field in the Universe after inflation has ended, and the Robertson-Walker (R-W) phase begins. As explained in ref. [12], the interpretation of this field as being quasi-classical is clear when thinking in terms of physical momenta and length scales, as opposed to comoving quantities. The amplitude of the fluctuations in this field, $\delta \chi^{2}$, on physical length scales $l$ in the $\mathrm{R}$-W Universe is given by

$$
\delta \chi^{2} \equiv \sigma^{2}=\frac{\mathrm{H}_{i}{ }^{2}}{4 \pi^{2}} \ln \left(\frac{l}{l_{\mathrm{c}}}\right)
$$

where $l_{\mathrm{c}}$ represents an effective ultra-violet cutoff on the physical momentum of the modes $\phi_{\mathrm{p}}$ which contribute to the amplitude in eq.(16). The formula for the dispersion $\sigma$ given in eq.(17) above, is clearly related to the t- dependent fluctuations (eq.(16)) produced during inflation. The origin of the t-dependence of the r.h.s. of that equation ultimately comes from modes with physical momenta $\mathrm{k} \sim \mathrm{H}_{i} \exp -\mathrm{H}_{i} \mathrm{t}$.

The explicit form of eq.(17) may be understood as follows. After inflation ends and the $\mathrm{R}-\mathrm{W}$ phase begins, new particle horizons appear, whose size are many orders of magnitude smaller than that of the 'blown-up' inflationary horizon. In fact the presently observed Universe can fit many times over inside the inflationary horizon. So we have to reconsider which modes contribute to the fluctuations in $\chi$ when we observe on a physical length scale $l$ which is bounded by the presently observed horizon. Obviously this last restriction means that we should only keep modes whose physical momentum k satisfies $2 \pi / \mathrm{k} \leq l$, so $l$ acts as an infrared cutoff on the spectrum of $\phi_{\mathrm{p}}$. At the other extreme, modes whose physical momentum is sufficiently large so that $2 \pi / \mathrm{k} \leq \mathrm{H}^{-1}$ have decaying amplitudes (here $\mathrm{H}$ is the Hubble constant during the $\mathrm{R}-\mathrm{W}$ phase ) and do not contribute significantly to the fluctuations. Therefore $l_{\mathrm{c}} \approx \mathrm{H}^{-1}$. By contrast, the modes whose wavelengths are greater than the $\mathrm{R}-\mathrm{W}$ horizon have constant amplitudes. All of these properties can be deduced by studying the time evolution of the $\phi_{\mathrm{p}}$ in $\mathrm{R}-\mathrm{W}$ space.

To summarize the above, the emergence of $\mathrm{R}-\mathrm{W}$ phase places effective cutoffs on the spectrum of $\phi_{\mathrm{p}}$. Taking these into account exactly reproduces the amplitude of fluctuations as given in eq.(17).

Before moving on to discuss the behaviour of these fluctuations at later times in the 
R-W Universe, let us comment on the possible values of the quantity $\bar{\chi}$.(The following arguments were originally used in discussing the mean value of the Peccei-Quinn axion field [13]). If the field $\chi$ had appeared after inflation, then microphysical processes would tend to smooth the field out over horizon volumes with, in general, different values of $\chi$ in different causally disconnected regions. (Here we are discussing an earlier epoch of the R-W phase when the cosine potential can be neglected). In this situation it would be natural to take $\bar{\chi}$ to be the r.m.s. of all the possible values between $-\pi$ and $+\pi$ namely $\chi_{\mathrm{rms}}=\pi / \sqrt{3}[13]$. If, on the other hand $\chi$ is present during inflation, (which is the case we consider in this paper), a quite different conclusion holds. The point is that a single inflationary patch easily encompasses our present Universe, so that the value of $\bar{\chi}$ in this patch is the one we see. But this value is actually arbitrary, because whilst the average of $\chi$ over all inflationary patches would again be the r.m.s. value $\pi / \sqrt{3}$, this says nothing about the value of $\chi$ in any one specific patch.

This result has important consequences for the formation of light domain walls via the fluctuations described previously. When we try to analyze the wall distribution in terms of percolation theory, $\bar{\chi}$ is related to the percolation probability $\wp[3]$ (to be discussed later). The fact that this is now arbitrary, leads to a richer (and more realistic) class of wall structures than had previously been thought possible [9].

Associated to the $\mathrm{R}-\mathrm{W}$ space fluctuations in $\chi$ there is a corresponding probability density $\mathrm{P}\left(\chi, \bar{\chi}, l / l_{\mathrm{c}}\right)$ given by

$$
\delta \chi^{2}=\left\langle(\chi-\bar{\chi})^{2}\right\rangle \equiv \int_{-\infty}^{+\infty} \mathrm{d} \chi(\chi-\bar{\chi})^{2} \mathrm{P}\left(\chi, \bar{\chi}, l / l_{\mathrm{c}}\right)
$$

which is just the Gaussian distribution of eq.(15) with de Sitter time t replaced by $\mathrm{H}_{i}{ }^{-1} \ln \left(l / l_{\mathrm{c}}\right)$. It is the distribution function in eq.(18) that is important in determining the probability of wall formation. In particular wall creation becomes significant if the dispersion $\sigma$ is comparable to $\mathrm{M}[3]$.

If one is to go beyond an estimation of the probability of wall formation, and discuss the kind of structures the walls create immediately after formation, statistical percolation theory seems to offer some hope. For example in [9], it was shown that assuming domain wall distribution can be described by that of percolation clusters in a percolating network, hierarchical matter clustering occurs. If matter clustering leads to galaxy formation, then percolation theory predicts galaxy clusters of specific richness (total number of galaxies) and distribution which fits fairly well with those catalogued by Abell [5]. 
Having said this, one really has to justify applying percolation theory to this problem. In 'two-colour' percolation, a lattice site (for example ) is occupied or unoccupied with probability $\wp$, and $1-\wp$ respectively. The analogue of this lattice in the cosmological case, is the R-W Universe at some time t, being filled with causally disconnected regions of size $\mathrm{H}^{-1}(\mathrm{t})$. Each of these regions contains a value of $\chi$ so that over the whole Universe, they are distributed according to the Gaussian $\mathrm{P}\left(\chi, \bar{\chi}, l / l_{\mathrm{c}}\right)$ of eq.(18). Clearly $\mathrm{P}\left(\chi, \bar{\chi}, l / l_{\mathrm{c}}\right)$, cannot as it stands, give a distribution of $\chi$ values which are just $\pm \pi$ everywhere. In the remaining part of this paper, we will show however, that this situation will change if we take into account the effect of the cosine potential on the values of $\chi$. We will then find that the Gaussian distribution will evolve into one which is non-Gaussian, displaying peaks near the two minima $\chi= \pm \pi$ of the potential. In this way we have a situation which is much closer to the one relevant to percolation theory.

To see how $\mathrm{P}$ can evolve into non-Gaussian form it is simpler to think of a discrete set of distinct random field values $\chi^{1}(\mathrm{t}), \ldots . \chi^{\mathrm{r}}(\mathrm{t})$, which occupy $\mathrm{N}$ causally separate regions at time t. ( $\mathrm{N}$ is like the lattice size in percolation models ). The distribution of such values will be given by a discrete version of the gaussian $\mathrm{P}$, so long as the values of $\chi^{\mathrm{s}}(\mathrm{t})$ for $\mathrm{s}=1$..r remain relatively constant in time. Since each $\chi^{\mathrm{s}}(\mathrm{t})$ satisfies an equation of motion like that in (1) with $\mathrm{V}$ given by eq.(8) and scale factor a(t) appropriate to the R-W Universe, the latter situation will be the case when the friction due to the Hubble expansion is greater than the acceleration on each $\chi^{\mathrm{s}}$ due to the cosine potential. That is, $\mathrm{P}$ will maintain its Gaussian form for $\mathrm{t}<\mathrm{t}_{0}$, where $\mathrm{t}_{0}$ is the time at which the $\chi^{\mathrm{s}}$ begin to roll down their potential. We shall refer to the values $\chi^{1}(\mathrm{t}), \ldots . \chi^{\mathrm{r}}(\mathrm{t})$ for $\mathrm{t}$ $<\mathrm{t}_{0}$ as $\chi_{0}^{1}, \ldots \chi_{0}^{\mathrm{r}}$. If $\mathrm{n}_{1}, \ldots \mathrm{n}_{\mathrm{r}}$ are the number of causal volumes occupied by the values $\chi_{0}^{1}, \ldots \chi_{0}^{\mathrm{r}}$ then the probability of finding the value $\chi_{0}^{\mathrm{s}}$ for $\mathrm{s}=1, . . \mathrm{r}$ is $\mathrm{n}_{\mathrm{s}} / \mathrm{N}$.

Now let us consider what happens when $t>t_{0}$. We have to consider solutions of eq.(1), with the boundary conditions $\chi^{\mathrm{s}}\left(\mathrm{t}=\mathrm{t}_{0}\right)=\chi_{0}^{\mathrm{s}}$ and $\dot{\chi}^{\mathrm{s}}\left(\mathrm{t}=\mathrm{t}_{0}\right)=0, \mathrm{~s}=1, . . \mathrm{r}$. The solutions $\chi^{1}(\mathrm{t}), . . \chi^{\mathrm{r}}(\mathrm{t})$ are trajectories with initial points $\chi_{0}^{1}, \ldots \chi_{0}^{\mathrm{r}}$, which in general, will cross each other at some time $t=t_{c}>t_{0}$ (whose value we shall discuss later) even if the initial values are all distinct. This is because the equation of motion for each $\chi^{\mathrm{s}}$ form a non-autonomous set of differential equations which depend explicitly on time t. However, for $t_{0}<\mathrm{t}<\mathrm{t}_{\mathrm{c}}$, the trajectories do not cross and we can approximate them as solutions to eq.(1) in the overdamped regime where the term involving second 
derivatives is dropped. The resulting equation is then easily seen to be autonomous, so this procedure is consistent.

The point is that since the trajectories $\chi^{\mathrm{s}}(\mathrm{t})$ are unique for $\mathrm{t}_{0}<\mathrm{t}<\mathrm{t}_{\mathrm{c}}$, the probability of finding the value $\chi^{\mathrm{s}}(\mathrm{t})$ will be the same as for $\mathrm{t}<\mathrm{t}_{0}$ i.e. for the value $\chi_{0}^{\mathrm{s}}$. This fact will allow us to determine the functional form of $\mathrm{P}_{\mathrm{t}}$ on $\chi\left(\chi_{0}, \mathrm{t}\right)$, where we now revert back to continuum notation, and $\mathrm{P}_{\mathrm{t}}$ denotes the distribution of the $\mathrm{t}$-dependent field $\chi\left(\chi_{0}, \mathrm{t}\right)$ for $\mathrm{t}_{0}<\mathrm{t}<\mathrm{t}_{\mathrm{c}}$. That is

$$
\mathrm{P}_{\mathrm{t}}\left(\chi\left(\chi_{0}, \mathrm{t}\right), \bar{\chi}_{0}, \sigma\right)=\mathrm{P}\left(\chi_{0}, \bar{\chi}_{0}, \sigma\right)
$$

Solving eq.(19) for $\mathrm{P}_{\mathrm{t}}$, we find

$$
\mathrm{P}_{\mathrm{t}}=\sqrt{\frac{2 \pi}{\sigma^{2}}} \exp \left(-\frac{2 \mathrm{M}^{2}}{\sigma^{2}}\left[\frac{\mathrm{f}\left(\chi\left(\chi_{0}, \mathrm{t}\right)\right)}{2 \mathrm{M}}-\frac{\bar{\chi}_{0}}{2 \mathrm{M}}\right]^{2}\right)
$$

where

$$
\mathrm{f}\left(\chi\left(\chi_{0}, \mathrm{t}\right)\right)=\chi_{0}
$$

i.e. $\mathrm{f}$ is the inverse function that maps the solution $\chi\left(\chi_{0}, \mathrm{t}\right) \rightarrow \chi_{0}$. Here we see the importance of requiring the trajectories be unique, otherwise the inverse mapping $\mathrm{f}$ would not exist for all initial values $\chi_{0}$.

Finally, we have to take care of the correct normalization of $\mathrm{P}_{\mathrm{t}}$. Eq.(19) was motivated from the discrete approach, whereas in passing to the corresponding continuum expressions we have to worry about Jacobian factors in going from $\mathrm{P}\left(\chi_{0}\right) \rightarrow \mathrm{P}_{\mathrm{t}}(\chi(\mathrm{t}))$. The latter involves changing variables from $\chi_{0}=\mathrm{f}(\chi(\mathrm{t}))$ to $\chi(\mathrm{t})$

$$
\int_{-\infty}^{\infty} \mathrm{d} \chi_{0} \mathrm{P}\left(\chi_{0}, \bar{\chi}_{0}, \sigma\right)=1=\int_{-\infty}^{\infty} \mathrm{d} \chi(\mathrm{t}) \frac{\mathrm{df}}{\mathrm{d} \chi(\mathrm{t})} \mathrm{P}_{\mathrm{t}}\left(\chi(\mathrm{t}), \bar{\chi}_{0}, \sigma\right)
$$

Hence, the correctly normalized continuum distribution is

$$
\hat{\mathrm{P}}_{\mathrm{t}}\left(\chi(\mathrm{t}), \bar{\chi}_{0}, \sigma\right)=\sqrt{\frac{2 \pi}{\sigma^{2}}} \frac{\mathrm{df}(\chi(\mathrm{t}))}{\mathrm{d} \chi(\mathrm{t})} \exp \left(-\frac{1}{2 \sigma^{2}}\left[\mathrm{f}(\chi(\mathrm{t}))-\bar{\chi}_{0}\right]^{2}\right)
$$

To see what $\hat{\mathrm{P}}_{\mathrm{t}}$ looks like, we need to solve eq.(1) in the overdamped approximation, and then compute the inverse function $\mathrm{f}$. The solution for $\chi(\mathrm{t})$ satisfying the boundary conditions discussed earlier, is 


$$
\begin{aligned}
& \chi(\mathrm{t})=2 \operatorname{Mtan}^{-1}\left(\tan \left(\frac{\chi_{0}}{2 \mathrm{M}}\right) \mathrm{e}^{\Delta\left(\mathrm{t}, \mathrm{t}_{0}\right)}\right) \\
& \Delta\left(\mathrm{t}, \mathrm{t}_{0}\right)=\frac{\mathrm{m}_{\chi}^{2}}{9}\left(\frac{1}{\mathrm{H}^{2}(\mathrm{t})}-\frac{1}{\mathrm{H}^{2}\left(\mathrm{t}_{0}\right)}\right)
\end{aligned}
$$

It is then straightforward to obtain the function $\mathrm{f}$, which gives the following $\hat{\mathrm{P}}_{\mathrm{t}}$

$$
\begin{aligned}
\hat{\mathrm{P}}_{\mathrm{t}}\left(\chi(\mathrm{t}), \bar{\chi}_{0}, \sigma\right) & =\sqrt{\frac{2 \pi}{\sigma^{2}}} \frac{\mathrm{e}^{-\Delta\left(\mathrm{t}, \mathrm{t}_{0}\right)}}{\left(\cos ^{2}\left(\frac{\chi(\mathrm{t})}{2 \mathrm{M}}\right)+\sin ^{2}\left(\frac{\chi(\mathrm{t})}{2 \mathrm{M}}\right) \mathrm{e}^{-2 \Delta\left(\mathrm{t}, \mathrm{t}_{0}\right)}\right)} \\
& \times \exp -\left(\frac{2 \pi}{\mathrm{M}^{2}}\left[\tan ^{-1}\left(\tan \left(\frac{\chi(\mathrm{t})}{2 \mathrm{M}}\right) \mathrm{e}^{-\Delta\left(\mathrm{t}, \mathrm{t}_{0}\right)}-\frac{\bar{\chi}_{0}}{2 \mathrm{M}}\right]^{2}\right)\right.
\end{aligned}
$$

Let us study the distribution $\hat{\mathrm{P}}_{\mathrm{t}}$ for the simple case when $\bar{\chi}_{0}=0$ i.e. when the original Gaussian is symmetric about $\chi_{0}=0$, as this will allow us to see the departure from Gaussian behaviour. We look first for the stationary points of $\hat{\mathrm{P}}_{\mathrm{t}}$, which satisfy

$$
\tan \left(\frac{\chi(\mathrm{t})}{2 \mathrm{M}}\right)=\tan \left[\frac{\left(\mathrm{e}^{\Delta}-\mathrm{e}^{-\Delta}\right)}{4 \mathrm{M}^{2}} \sigma^{2} \sin \left(\frac{\chi(\mathrm{t})}{\mathrm{M}}\right)\right] \mathrm{e}^{\Delta}
$$

Obvious solutions to eq.(27) are $\chi=0 \bmod 2 \pi$, but there are other solutions that depend on $\Delta$ but which are difficult to find analytically. However, let us concentrate on the central stationary point at $\chi=0$. One can show that

$$
\operatorname{sign}\left(\frac{\mathrm{d}^{2} \hat{\mathrm{P}}_{\mathrm{t}}}{\mathrm{d} \chi^{2}}\right)_{\chi=0}=\operatorname{sign}\left(\frac{\mathrm{e}^{\Delta}-\mathrm{e}^{-\Delta}}{2 \mathrm{M}^{2}}-\frac{1}{\sigma^{2}}\right)
$$

Thus, at $\mathrm{t}=\mathrm{t}_{0}, \Delta=0$ and the point $\chi_{0}=0$ is clearly a maximum, as it should be since $\mathrm{P}$ is a Gaussian centred at zero. As $\Delta$ increases with time, this situation continues until a critical value $t_{\text {crit }}$ is reached, which is defined by

$$
\mathrm{e}^{\Delta_{\text {crit }}}-\mathrm{e}^{-\Delta_{\text {crit }}}=\frac{2 \mathrm{M}^{2}}{\sigma^{2}}
$$

When $\Delta>\Delta_{\text {crit }}, \chi=0$ changes from a maximum to a minimum. Now, on the other hand, $\hat{\mathrm{P}}_{\mathrm{t}}$ is a bounded function as $\chi(\mathrm{t}) \rightarrow \pm \infty$ hence if there is a minimum at $\chi=0$ there must exist other maxima (at least 2 ) at some non-zero values of $\chi$. We expect that similar conclusion holds for distributions with $\bar{\chi} \neq 0$, which although difficult to prove analytically, is verified by plots of $\hat{\mathrm{P}}_{\mathrm{t}}$ (see figs.3 ). 
Before discussing these numerical plots, we should return to the issue concerning the existence of the crossing time $t_{c}$ beyond which trajectories $\chi\left(\chi_{0}, t\right)$ with different initial values $\chi_{0}$, cross. In fig.1, numerical solutions to the full equations of motion are plotted as solid curves for various initial values $\chi_{0}$. It is clear from these, that $t_{c}$ exists, and is approximately given as $\mathrm{m}_{\chi} \mathrm{t}_{\mathrm{c}} \approx 3$. Note we have not considered initial conditions beyond $\chi_{0} / \mathrm{M}= \pm \pi$ because $\hat{\mathrm{P}}_{\mathrm{t}}$ is exponentially suppressed in this regime. By contrast, the dashed curves in fig.2 are the analytic solutions given in eqs. $(24,25)$. We see that for $\mathrm{m}_{\chi} \mathrm{t} \leq \mathrm{O}(1)$, the latter are a good approximation to the numerical solutions of the complete equations of motion. Thus, the expression for $\hat{\mathrm{P}}_{\mathrm{t}}$ of eq.(26) is only correct for times up to maximum $\mathrm{t}_{\max }$, with $\mathrm{m}_{\chi} \mathrm{t}_{\max } \approx \mathrm{O}(1)$.

In figs.3a-3d we have plotted the distribution $\mathrm{P}_{0} \equiv \hat{\mathrm{P}}_{\mathrm{t}} / \sqrt{2 \pi / \sigma^{2}}$ for values of $(\bar{\chi}, \sigma / \pi \mathrm{M})$ corresponding to $(0,0.5),(0,0.8),(\pi / 4,0.5)$ and $(\pi / 4,0.8)$ respectively. The plots are for time $\mathrm{t} \approx \sqrt{2} \mathrm{~m}_{\chi}{ }^{-1}$. The values of $\sigma / \pi \mathrm{M}$ are chosen so as to display the double maxima in $\hat{\mathrm{P}}_{\mathrm{t}}$, described earlier. Such values are very reasonable, because as was shown in ref. [3], the probability of domain walls forming in these models can be estimated as the ratio $\sigma / \pi \mathrm{M}$. The dashed curves in figs.3 are those of the original Gaussian distribution at $\mathrm{t}=\mathrm{t}_{0}$. It is clear from these plots that the effect of the cosine potential is to focus probability into the minima of the potential at $\chi= \pm \pi$, and we can see roughly that this probability is equal to the area under the dashed curve to the right or left of the point $\chi=\bar{\chi}_{0}$. These two areas play the role of the probability $\wp$ and $(1-\wp)$ in percolation theory.

In conclusion, we have shown how the probability distribution $\mathrm{P}(\chi, \bar{\chi})$ generated during inflation evolves from Gaussian to non-Gaussian behaviour during the R-W phase. This was a consequence of the cosine potential of $\chi$ focussing probability into the two minima at $\chi= \pm \pi$. The amount of focussing in each minima depends on the value of $\bar{\chi}$. These results justify the percolative approach to domain wall formation in this model, with the important consequence that the percolation probability $\wp$ can take any value $0<\wp<1$.

\section{Acknowledgements}

We would like to thank Burt Ovrut for many useful discussions, and past collaborations in this subject. 


\section{References}

[1] C. Hill, D. Schramm and J. Fry, Comm. on Nucl. and Part. Phys. 19 (1989) 25;

C. Hill, D. Schramm and D. Widrow, Fermilab preprint -PUB-89/166-T (1989).

[2] C. Hill and G.Ross, Nucl.Phys. B311 (1988) 253, Phys. Lett. 203B (1988) 125; A. Gupta, C. Hill, R. Holman and E. Kolb, Phys. Rev. D45 (1992) 441; J. Frieman, C. Hill and R. Watkins, Fermilab-PUB-91-324-A(1991).

[3] B.A. Ovrut and S. Thomas, Phys. Lett. 277B (1992), 53.

[4] A. Linde and D.H. Lyth, Phys. Lett.246B (1990) 353; D. H. Lyth, Phys.Lett. (1991) .

[5] G. Abell Astrophys. J. Suppl.3(1958) 211.

[6] N. A. Bahcall, Astrophys. J. 232 (1979) 689.

[7] V. de Lapparent, M. J. Geller and J. Huchra, Astrophys. J. 302,L1 (1986) 1986.

[8] G. F. Smoot et al, Astrophys. J.(1992) TBD.

[9] Z. Lalak, B. A. Ovrut and S. Thomas, University of Pennsylvania preprint UPR0507T; Z. Lalak and B.A. Ovrut UPR-0504T (1992).

[10] For example see the review by D. Stauffer, Phys. Rep. 54 (1979) 1.

[11] A.A. Starobinsky, Phys. Lett.117B (1982) 175, also in 'Current Topics in Field Theory, Quantum Gravity, and Strings,' Lecture Notes in Physics, ed H.J. DeVega and N. Sanchez, vol 246 (Springer Heidelberg, 1986) p. 107; A. Goncherov and A. Linde,Sov. Phys. JETP 65 (1987) 635; S.-J. Rey, Nucl. Phys. B284 (1987) 706.

[12] 'Particle Physics and Inflationary Cosmology' by A. Linde, Contemporary Concepts in Physics vol 5, (Harwood Academic Pubs. 1990) page 161.

[13] See for example 'The Early Universe' by E. Kolb and M.S. Turner, (AddisonWesley Publishing, 1990) page 431 for a discussion of this point. 


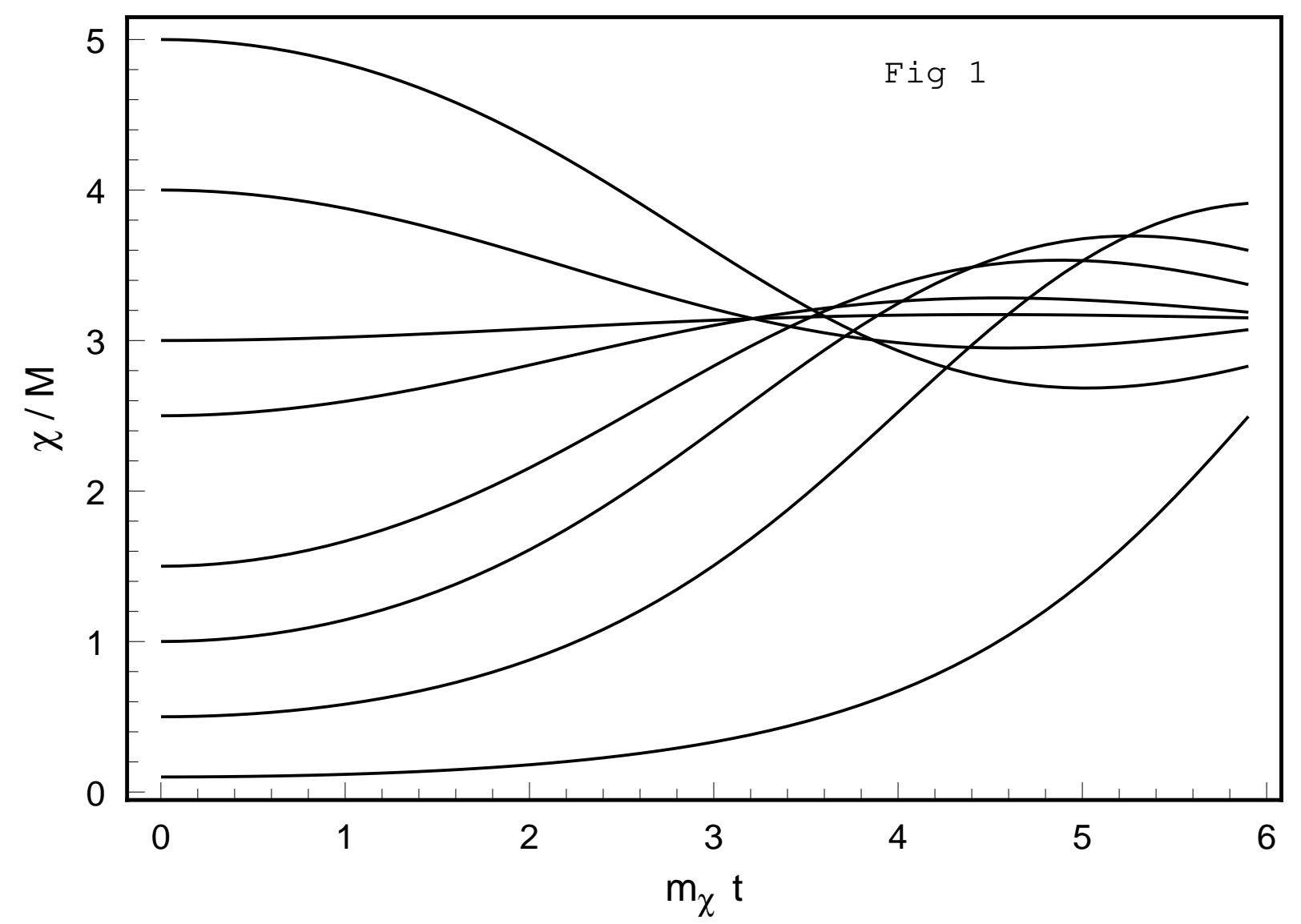




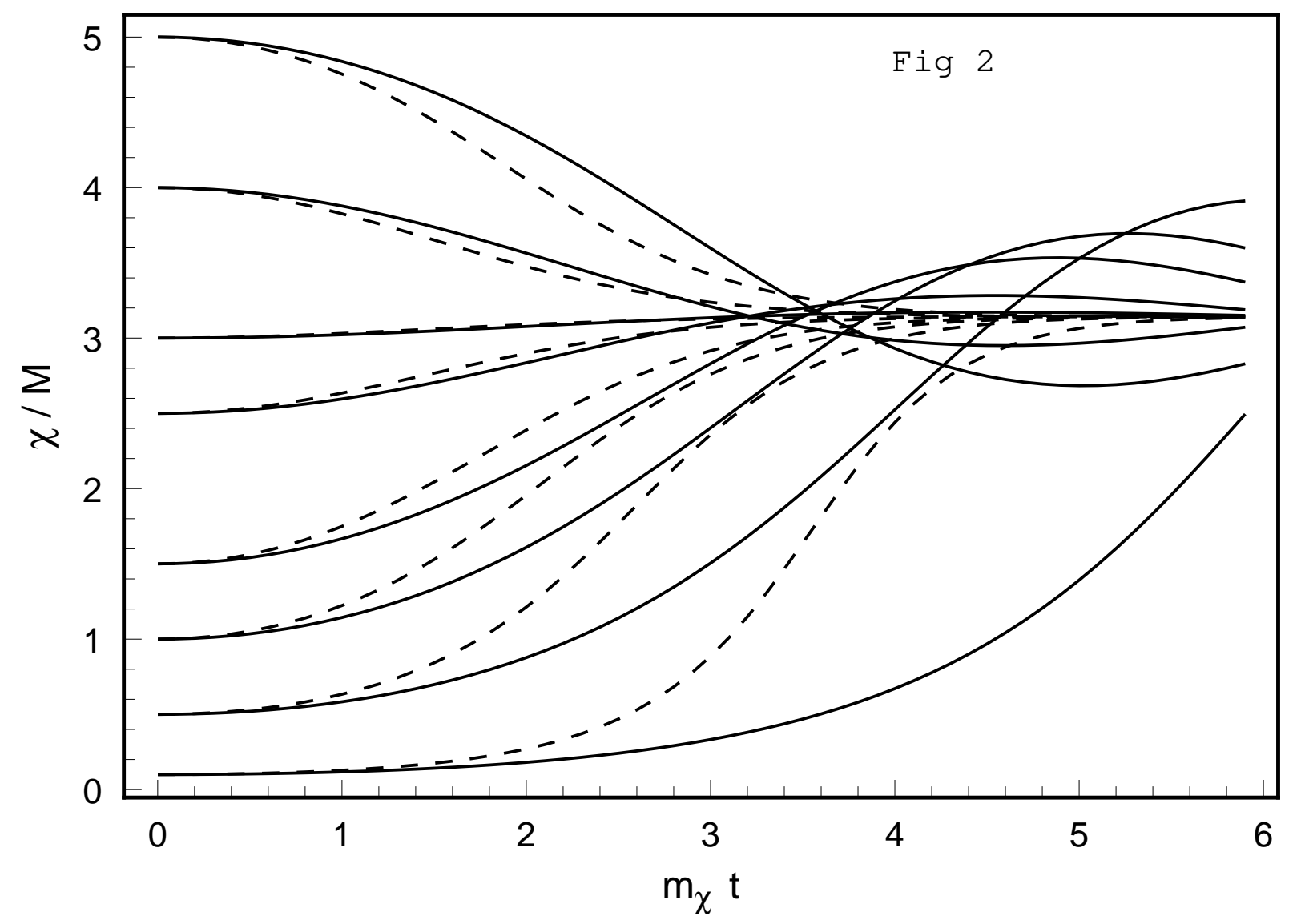




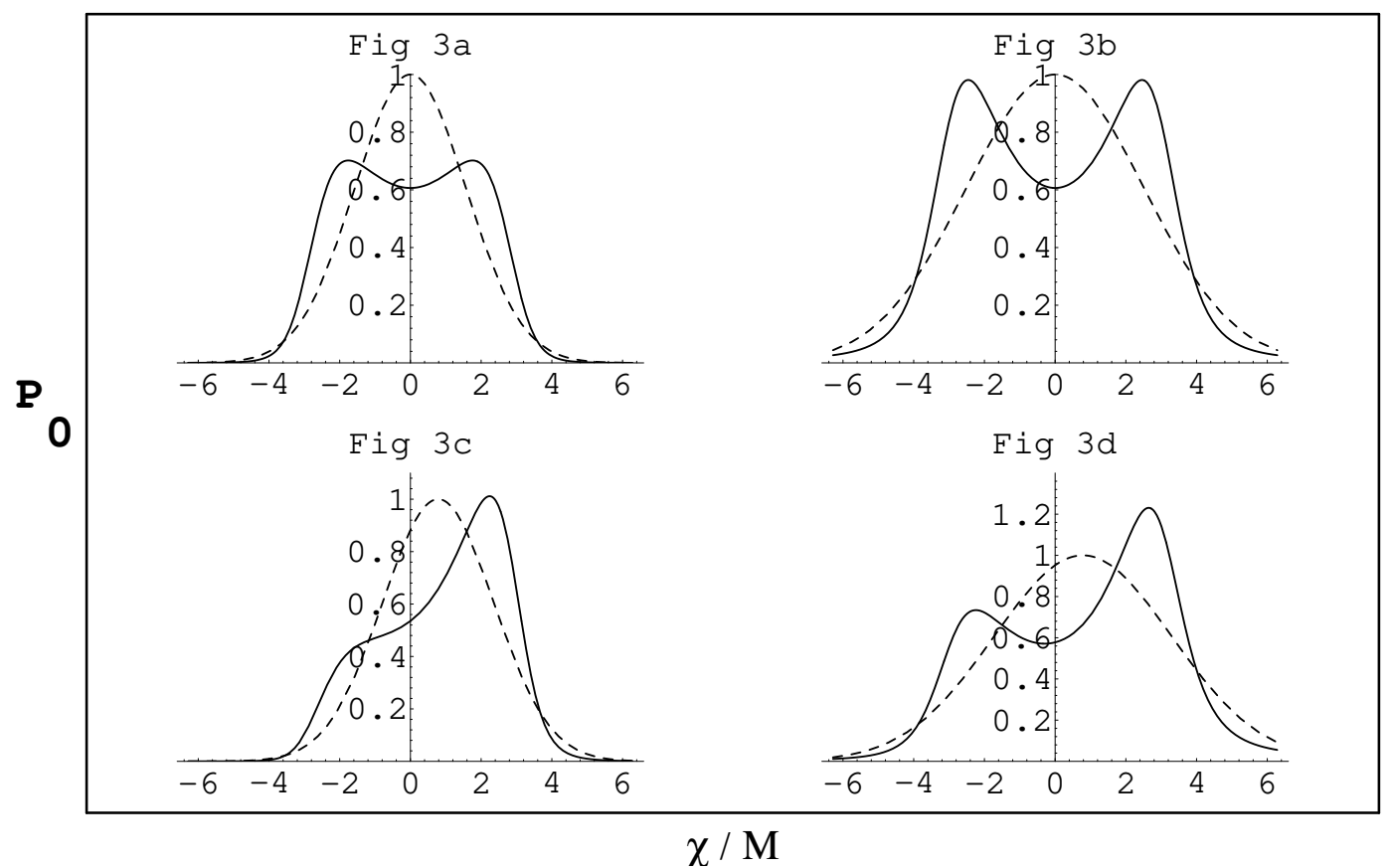

